\title{
Maturation processes in automatic change detection as revealed by event-related brain potentials and dipole source localization: significance for adult AD/HD
}

\author{
Nele Wild-Wall, Robert D. Oades, Stephanie A. Juran.
}

Biopsychology Group, University Clinic for Child and Adolescent Psychiatry, Virchowstr. 174, 45147, Essen, Germany

2005 International Journal of Psychophysiology, 58, 34-46

\begin{abstract}
:
Mismatch negativity (MMN) is an event-related potential reflecting automatic attention-related information processing marking the detection of auditory change. The bilateral scalp distribution develops by 14 years of age, and is elicited with adult latencies by 17 years. But consistent with reports of continued brain maturation after adolescence we show here that features of the temporal and frontal lobe dipole sources also continue to develop in the third decade of life. This has consequences for studies of the developmental course of MMN anomalies reported in childhood attention-deficit/hyperactivity disorder into adulthood. Two groups of healthy subjects with mean ages of 17 and 30 years were presented with a 3-tone auditory oddball. The duration-deviant $\mathrm{MMN}$ was recorded during attention to a visual discrimination (auditory passive condition) and an active auditory discrimination. MMN amplitudes were smaller in the older subjects and the MMN lasted longer over the right hemisphere. Latencies and moments of the 4 dipoles in the temporal and frontal lobes did not distinguish the two subject-groups. But both temporal lobe sources were located significantly more ventrally and further left in the young adult than in the adolescent subjects. The left cingular source moved posteriorly and the right inferior frontal source moved antero-medially in the older subjects. Brain development in the third decade may cause the two frontal sources to move apart on the rostro-caudal axis but the temporal lobe sources to move left on the lateral and down on the dorso-ventral axes. Thus special care is necessary in interpreting putative dysfunctional neurobiological changes in developmental attention deficit disorders where as yet unspecified subgroups may show a late developmental lag, partial lag or no lag at all associated with other impairments.
\end{abstract}

Key words: Attention-deficit; Development; Dipole; Frontal lobe; Maturation; Mismatch Negativity; Temporal lobe;

Correspondence: Robert D. Oades, University Clinic for Child and Adolescent Psychiatry, Virchowstr. 174, 45147 Essen, Germany.

Acknowledgments: NWW was supported by the Alfried Krupp von Bohlen und Halbach Stiftung, and SAJ was supported by University of Duisburg-Essen IFORES funds.

\section{Introduction}

It is an intriguing issue to determine the nature and prevalence of functional impairments in adult manifestations of attentiondeficit/hyperactivity disorder (AD/HD). What features of $A D / H D$ as a child and adolescent a) remain, b) get worse and c) disappear in young adults with an apparent residual disorder? Are there other problems which appear de novo, or appear with a different quality compared to the difficulties experienced earlier in development?

For example, for about a third of those originally diagnosed with $A D / H D$, by the end of the second decade of life, typically some cognitive impulsivity and distractibility may remain, while self-esteem, academic and social abilities may get worse, but hyperactivity may improve (Hendren, 1991; Willoughby, 2003). Neuropsychological testing of adults with AD/HD has shown poor inhibitory abilities on the stoptask (Murphy, 2002), inattention (Biederman et al., 2000) and auditory distractibility (Corbett and Stanczak, 1999). In turn these results implicate right inferior frontal function (Aron et al., 2003) and poor auditory cortex (temporal lobe) modulation of information processing. It is thus 
of particular interest whether markers of the early stages of sensory processing, for automatic attention $^{1}$ to and controlled processing of audiovisual stimuli (that involve the participation of these brain regions [see below]), show some persistent differences in adult manifestations of $A D / H D$. Astonishingly there is very little electrophysiological, structural or functional evidence on this issue even for normal postadolescent development (Casey et al., 1997).

In childhood $A D / H D$ the nature of the excitation of the ascending pathways and the arrival of auditory information in the sensory cortices marked by the N1 evoked potential is unremarkable in size or source characteristics (Oades et al., 1996; Kemner et al., 2004). However, the normal differential amplitude of N1 elicited by relevant vs. irrelevant stimuli is less distinct (review, Barry et al., 2003). The differences between children with $A D / H D$ and their healthy counterparts become more marked for the succeeding stages of processing reflected by the P2 and N2 auditory event-related potentials (ERPs) that occur either side of the threshold for stimuli reaching conscious perception (Libet, 2002). These ERPs mark respectively stages of information processing that reflect early automatic (latency < ca. $200 \mathrm{~ms}$ ), and later controlled information processing (latency $>$ ca. $200 \mathrm{~ms}$ ). The P2 may represent the inhibition of the influence of stimuli that might otherwise interfere with further processing of salient and potentially important stimuli (Oades et al., 1996; Oades, 1998) and the N2 indexes the categorization of stimuli prior to the updating of memory templates (Ritter et al., 1983; Lange et al., 1998). The central P2 and frontal N2 scalp distributions do not appear until the end of adolescence (Oades et al., 1997). P2 amplitudes are often larger in childhood AD/HD (Oades et al., 1996) and perhaps more so by the end of adolescence (Johnstone et al., 1996). In contrast $\mathrm{N} 2$ peaks in $\mathrm{AD} / \mathrm{HD}$ may be smaller (or show less differentiation between stimuli), and may not take up a frontal topography by the end of adolescence (Johnstone and Barry, 1996; Johnstone et al., 1996; Lazzaro et al., 2001). Sources for P2 activity lie in the secondary association cortices of the temporal lobe (Jückel

\footnotetext{
1 Attention is defined as the selective aspect of perception (Treisman, 1969), and may be divided into preconscious automatic, and later controlled processes (Schneider and Shiffrin, 1977)
}

et al., 2003), and for N2 in the posterior part of the anterior cingulate and in connected prefrontal regions (Mathalon et al., 2003).

Clearly while the characteristics of the P2 and N2 ERPs and processing at these early stages on either side of the threshold for conscious perception are abnormal in AD/HD children, their sources in the brain, at least for $\mathrm{P} 2$ are apparently normal (Kemner et al., 2004). However, these are precisely the locations in which the four dipole sources underlying auditory change detection and the associated involuntary switch of attention-related processing (mismatch negativity, MMN) are found (Jemel et al., 2002). Thus the study of the MMN dipoles, reflecting preconscious attentional selection of auditory information, and of alterations that may occur in the regions supporting these sources becomes import-ant. These regions are also those in which neuropsychological function seems disturbed (above), and those where the development in $A D / H D$ of $M M N$ and cognitive inhibitory functions have been reported (ERP-study, Oades et al., 1996; fMRI study, Rubia et al., 2001). The study of Oades and colleagues (1996, also Rothenberger et al., 2000) found that instead of the MMN topography developing as usual over right frontal areas, it developed on the left, implicating a reversal of the asymmetric development of MMN. A similar result was reported for the N2 (Pliszka et al., 2000). Thus although the characteristics of the MMN recorded at the scalp appear broadly normal (Oades et al., 1996; Winsberg et al., 1998) or modestly reduced in the midline (Kemner et al., 1996; Rothenberger et al., 2000) its topography indicates something unusual in its generation. $A$ close study of MMN source development is necessary, because of the complexities surrounding the progression of $A D / H D$ into adulthood (start of the introduction) and the major recent advances in understanding normal brain development, especially from late adolescence. So we decided a close study of normal adolescent/ post-adolescent development would be a wise precaution using MMN as the marker.

What is known about post-adolescent structural development? Until recently most studies of the maturation of the brain in terms of regional proliferation (morphology, Thatcher, 1987), cognitive function (e.g. problem-solving and abstract thinking, Goldman-Rakic, 1987), and 
neuronal activity (e.g. decreasing frontal theta/alpha ratios in the electroencephalogram, Matousek and Petersen, 1973; Duffy et al., 1984) suggested that the processes were broadly complete by the end of adolescence. Often this reflected simply not looking for changes in the third decade of life. The main exception was the continued extension of myelination through the third decade of life (Yakovlev and Lecours, 1967; Blinkov and Glezer, 1968). This continued development is of major importance for the fibre tracts linking the hemispheres and for interhemispheric control. Indeed development may continue even longer than the third decade in the corpus callosum (Benes et al., 1994), especially in the middle and posterior portions (Giedd, 1997). This in turn has implications for the maturation of the control of affect, anxiety and sociability (Benes et al., 1994). Further, related to continued white-matter development and the longestablished catecholamine involvement in $A D / H D$, it has also been recognised in rodents and monkeys that fibre length and density in the ascending dopaminergic pathways increase in adolescence, and decrease thereafter (Kalsbeek, 1989; Rosenberg and Lewis, 1994). Continued change in arborization patterns and spine density (Giedd, 1997), especially in frontal regions (Lewis et al., 1995) imply potential changes of dopamine receptor densities (Unis et al., 1995) and catecholaminergic control of 'higher functions' in young adults.

Currently it is recognized that major indices of cortical development are represented by measures of increased surface area and increasing white- to grey-matter ratios. (Grey matter volumes contribute to this ratio by decreasing somewhat.) Development spreads in waves from dorsal parietal regions such that primitive limbic and sensory regions mature early, post-pubertally. Within the frontal and temporal lobes the last regions to develop are those that evolved most recently. These are the anterior inferior- to superior prefrontal cortex and the heteromodal posterior superior temporal gyrus (Sowell et al., 2001; Gogtay et al., 2004). These are the structures that continue to develop in the post-adolescent period. They are also the regions in which attention-related processing will have a strong influence on function and where anomalies may show up in adult manifestations of attention-deficit.

The advances over the last 10 years in understanding the normal development of brain anatomy over the third decade of life have not been matched by studies of cognitive development. Least of all have there been any attempts to relate the post-adolescent development of cognitive processes to the underlying changing neurobiological bases (see Casey et al., 1997). Thus, as the sources for MMN activity appear close to or within some of the areas mentioned, our aims in this study were to investigate the dipole sources for auditory change detection in normal persons in the lateand post-adolescent periods to see if there were developmental changes matching structural maturational processes. To our knowledge, there are no psychophysiological studies about how normal frontal or temporal lobe maturation in the third decade of life might affect automatic information processing. In the context of AD/HD, information on the changes in the characteristics of the biological bases for an automatic attentional process in normal subjects will inform on the controversial contribution of a developmental lag or of some other aetiology for their cognitive problems. Future studies may not take account of the differences occurring between adolescence and adult-hood in the interpretation of results from adult $A D / H D$ patients vs. controls if they are merely matched for age. For, as stated, different features may remain, get worse, disappear or appear de novo. Based on the results of the current investigation it will be possible to look for sub-groups whose problems derive from different diatheses.

\section{Methods and Materials}

\section{Subjects}

Data were analysed from 40 subjects separated into 2 age groups. In the adolescent group were 22 healthy subjects with a mean age of 17.6 years (SD 0.4, 17Y-group: 12 male and 10 female). In the young adult group were 18 healthy subjects with a mean age of 30.4 years (SD 1.4, 30Y-group: 7 male and 11 female). They were recruited by advertisement and paid for participation. None of the subjects had current psychiatric or psychological problems (SCIDII) or a history of neurological or psychiatric disorders, nor did they use drugs affecting the central nervous system. The two groups did not differ on socio-economic ratings based on parental profession (mean $3.0 \pm$ 0.5 on a scale of 6; Erikson and Goldthorpe, 1992). The groups were also comparable on the results of the short IQ test that consisted of the 
information, arithmetic, digit-symbol, blockdesign subtests which correlate well with fullscale measures (17Y: 115.8, SD 4.2; 30Y: 109.9 SD 4.0: Blyler et al., 2000). All had normal or corrected to normal vision and normal hearing (thresholds $<30 \mathrm{~dB}$ : BCA audiometer).

\section{Stimulus presentation}

A 3-tone auditory oddball was presented binaurally using sinusoidal tones (76 dB SPL). Standard tones had a frequency of $800 \mathrm{~Hz}(p=$ $80 \%$, duration $80 \mathrm{~ms}$ including $10 \mathrm{~ms}$ rise and fall times), frequency-deviants had the same duration but a frequency of $600 \mathrm{~Hz}(p=10 \%)$ and duration deviants had the frequency of the standards but lasted only $40 \mathrm{~ms}(p=10 \%)$. The stimulus onset asynchrony (SOA) varied from 850-1050 ms (mean $950 \mathrm{~ms}$ ). The order of stimulus presentation was pseudo-random with each deviant preceded by at least one standard.

Four auditory trial blocks were run during a simple visual red or green circle discrimination (200 tones in each block). The red and green circles (50:50) subtended an angle of $3.8^{\circ}$ at 1.5 $\mathrm{m}$ and changed at random every $1100 \mathrm{~ms}$ on a PC screen. Subjects responded in the passive condition alternately between blocks with the left and right hand to the green circle. After a short pause the audiovisual presentation was repeated with response required to the frequency-deviant tone. Auditory stimulus detection is not suppressed during various concurrent visual processes (Harris and Lieberman, 1996), the onsets of the visual and auditory stimuli were controlled so that they did not coincide. This presentation permitted the calculation of the MMN response to the duration deviant in states where attention was directed to another modality (the first auditory-passive condition) and to the same modality (the second, auditoryactive condition).

\section{Data recording}

The EEG was recorded (Neuroscan, El Paso, $\mathrm{TX}$ ) from 31 electrode sites including $\mathrm{Fpz}, \mathrm{Fz}, \mathrm{Cz}$, $\mathrm{Pz}, \mathrm{Oz}, \mathrm{FCz}, \mathrm{CPz}, \mathrm{F} 3, \mathrm{~F} 7, \mathrm{~F} 4, \mathrm{~F} 8, \mathrm{FT7}, \mathrm{FT} 8, \mathrm{C} 3, \mathrm{C} 4$, P3, P4, CP3, CP4, T3, T4, T5, T6, TP7, TP8, M1, and $M 2$ according to the modified 10-20 International System (Pivik et al., 1993). Tin electrodes in an electrode cap (Electro-Cap International Inc.) were used and impedance kept below $5 \mathrm{k} \Omega$. A vertical EOG was recorded from the supra-orbital ridge of the right eye and a horizontal EOG from the outer canthus of the right and left eye to monitor blink- and eye-movements for offline rejection of artefacts $(>50 \mu \mathrm{V})$ in EOG leads. All electrodes were referenced to linked earlobe electrodes. The average-reference was recomputed off line. A band-pass filter was set at 0.1-100 Hz. Data were digitised with 16-bit resolution, sampled at $500 \mathrm{~Hz}$ and stored on a hard disk. Records were epoched separately for each tone type with $100 \mathrm{~ms}$ pre-stimulus baseline. Offline, a $30 \mathrm{~Hz}$ low-pass (24-dB/octave) was applied. ERPs were based on 63-68 artefactfree trials and $>600$ trials for the standards.

During the study electrode positions relative to the nasion, left and right periauricular skull landmarks were digitised using ultrasound to control for individual head shapes (Zebris, Munich) and to improve the accuracy of source analysis. To illustrate these sources we use one of the high resolution $\mathrm{T1}$-weighted $3 \mathrm{D}$ anatomical images recorded from the subjects with a Siemens 1.5-T sonata (Munich, Germany: with an MP RAGE sequence using a $384 \times 384 \times 192$ matrix giving a resolution of $0.5 \times 0.5 \times 1.0 \mathrm{~mm}$, repetition time/echo time/ flip angle $=30 \mathrm{~ms} / 10$ $\mathrm{ms} / 30^{\circ}$ with a $256 \mathrm{~mm}$ field of view).

\section{Data analysis}

In both the visual (auditory passive condition) and the auditory (active condition) modality reaction times and percentage of hits were compared between groups with two-tailed ttests.

The MMN was calculated by subtracting the ERPs to standard tones from those elicited by the duration-deviant. It was defined by a negativegoing peak between 90 and 225 ms after stimulus onset. The MMN was averaged and analysed separately for the passive and active experimental conditions and the two groups (17Y- and 30Y-groups). For the averagereferenced data the mean activation in $30 \mathrm{~ms}$ windows was investigated. We restricted analysis to the consecutive intervals $135-165$ and 165-195 $\mathrm{ms}$ where inspection of the waveforms showed there to be potential group differences (Figure 1). These differences were explored with a repeated measures analysis of variance (ANOVA) including all 29 scalp electrodes and the two subject groups. For all ANOVAs the Greenhouse- 


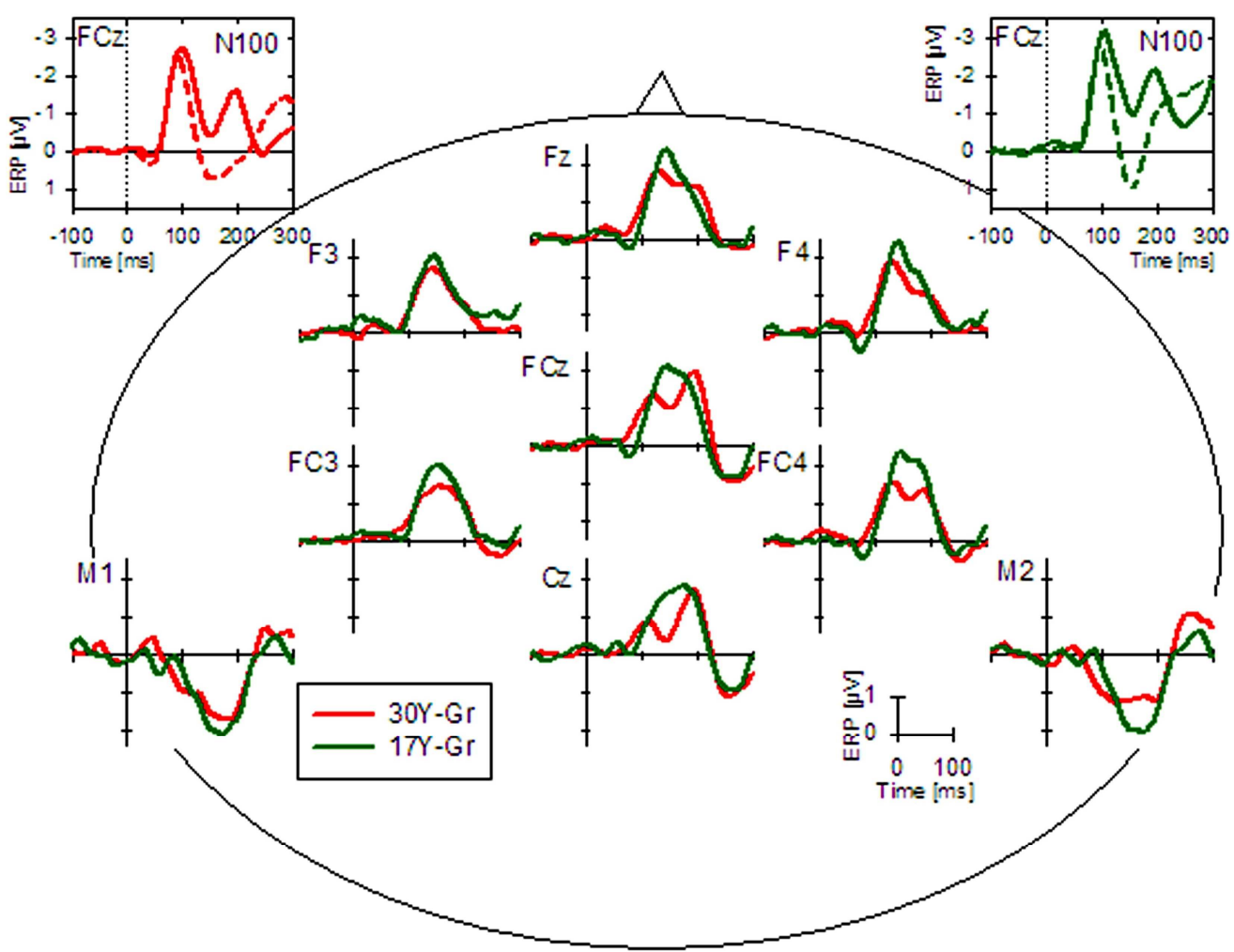

Figure 1: The central part of the figure illustrates the duration deviant MMN waveforms for the 17Y group and the $30 \mathrm{Y}$ group of subjects recorded from 7 fronto-central and two mastoid electrode-sites during the passive auditory condition. The inserts show the N1 waveforms elicited by the standard and the duration deviant auditory stimuli in the adolescent (top right) and young adult subject groups (top left).

Geisser epsilon was used as a conservative correction for the degrees of freedom. The same analyses were again calculated with vector scaled data (McCarthy and Wood, 1985) in order to ascribe differences between groups either to amplitude changes or to topographical changes. (MMN peak latencies were analysed separately from the linked-earlobe data by ANOVA.) This was followed by a detailed analysis of the data from 2 mastoid and 7 fronto-midline recording sites (F3, Fz, F4, FC3, FCz, FC4, Cz, M1, M2) and the two subject groups to reduce the degrees of freedom. The locus of effect was determined with two-tailed t-tests.

Brain electrical source analysis (BESA, [Scherg and Berg, 1991]) for modelling MMN equivalent dipoles was based on average-referenced ERPs in a time window from $20 \mathrm{~ms}$ before to $40 \mathrm{~ms}$ after the respective MMN peak, first in the grand mean, and second in the individual waveforms. BESA source-modelling allows iterative fitting of the dipole location and orientation in a spherical head-model with an inner radius of $85 \mathrm{~mm}$ corrected for brain, skull, and scalp conductivity
(Scherg and Picton, 1991), until the difference between the recorded surface data in the timewindow and the calculated surface data of the dipole-model is minimised (least square fit). The goodness of fit is expressed by the mean residual variance (RV), and the best fit is the lowest RV not explained by the model, as a percentage of the signal variance within the given time window.

As a starting point we used the MMN fourdipole-model as proposed by Jemel et al. (2002). Bilateral frontal and temporal lobe dipoles were necessary to explain $>95 \%$ of the topographic variance. For both groups the four-dipole model was fitted separately to the ERP grand mean that describes the most reliable characteristics of the spatio-temporal data matrix. During the iterative best-fitting estimations the stronger temporal followed by the less strong frontal lobe dipoles were initially constrained to the hypothesis and then allowed to move within the model brain without constraint. In the final unconstrained 

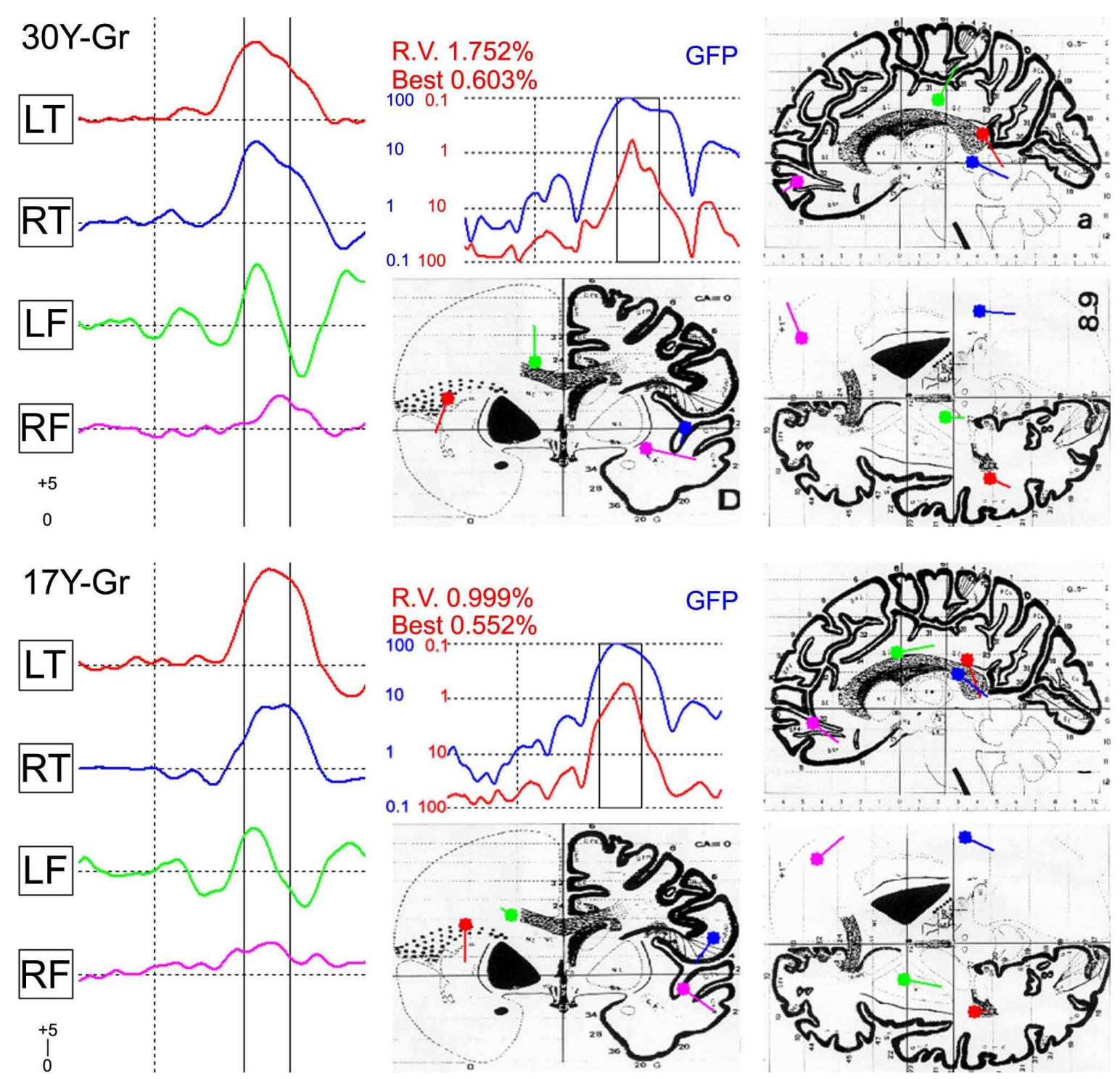

Figure 2: On the left of the figure is the time course for activity of the four dipole moments whose location is marked in the frontal and temporal lobes on axial, coronal and sagittal sections of the modified Montreal brain atlas in the middle and on the right of the figure. The residual variance (RV) and best fit for the BESAcalculated group dipole solution is plotted in the middle. GFP = global field power.

solution (with an acceptable criterion of RV $<2 \%$, Jemel et al., 2002) the temporal lobe sources were mildly and the frontal lobe sources markedly asymmetric.

Stereotaxic Talairach coordinates were calculated for these sources with software developed by Garnero et al. (Crottaz-Herbette and Ragot, 2000). This allowed placement of the solutions on the modified Montreal Neurological Institute brain atlas. In order to calculate statistical differences between groups individual source solutions were re-calculated for each subject based on the respective group solution as a starting point. Individual MMN waveforms were additionally high- (1.5 Hz, 6db/octave) and low pass filtered ( $15 \mathrm{~Hz}, 12 \mathrm{db} /$ octave). If a criterion of $\mathrm{RV}<4 \%$ was not reached without leaving the main hypothesis of the 4-dipole-model (Jemel et al., 2002) the subject was discarded from statistical analysis of the dipoles. Two 17Y- and three 30Y-group subjects were removed. This relaxed RV threshold was used in order to account for the decreased signal-to-noise ratio of a single subject's waveforms contributing to the individual solution. The distances of the individual best solutions from the mean of the individual solutions were mapped on to the brain image of a subject from the 30Y-group 
normalised with reference to the SPM99 T1-brain template (Wellcome Department of Cognitive Neurology, University College London) with the radius of the circle or ellipse representing the standard deviation from the mean location of the individual solutions.

The locations of all four dipoles were compared between the two groups with a multivariate ANOVA including the four-level factor dipole as well as the between-subject factor group. Post-hoc F-Tests were calculated for comparison of the $\mathrm{x}, \mathrm{y}$, and z-coordinates of each dipole. Group-dependent differences of dipole peak strength, and peak latencies were determined by univariate ANOVAs. Here, a twolevel between-subject factor (17Y- and 30Ygroup) was included as well as a four-level withinsubject factor dipole (left and right, frontal and temporal lobe locations).

\section{Results \\ Performance data}

There were no differences either for response time in the visual vigilance control test between adolescent and young adult subjects in the auditory passive condition ( $\mathrm{t}<1$; $496 \mathrm{~ms}$, SD 125 vs. $467 \mathrm{~ms}, \mathrm{SD} 82$ ) nor for percentage of visual hits $(\mathrm{t}(36)=-1.3, \mathrm{p}=0.19 ; 97.2 \%$, SD 7.8 vs. $99.7 \%$, SD 0.6). The same holds true for the frequency deviance detection in the auditory active condition (auditory RT: $\mathrm{t}<1,409 \mathrm{~ms}$, SD 61.4 vs. $401 \mathrm{~ms}$, SD 61.5; auditory percentage of hits: $\mathrm{t}<1,90.3 \%$, SD 11.1 vs. $92.4 \%$, SD 11.9).

\section{Scalp Recordings of Event-related Potentials (ERPS)}

As can be seen in figure 1 (inserts), N1 amplitudes in response to standard and deviant stimuli were very similar and did not differ significantly $(F<1.0)$. MMN peak latencies were non-significantly shorter in the adolescent $(F C z$, $155 \mathrm{~ms}$ SD 20) than in the young adult subjects (166 ms SD 26, $F<1.0$ ). Preliminary analyses with all electrodes showed that significant amplitude differences existed in the $30 \mathrm{~ms}$ time window around the peak latency of the MMN (135-165 ms post stimulus: $F_{28,1064}=2.8, P=0.02, \varepsilon=0.18$ ). Repeating the analyses (group $\mathrm{x}$ electrode interaction) with vector-normed data did not give a significant result (e.g. $F_{28,1064}=1.7, P=0.18$, $\varepsilon=0.18$ ), which suggested that amplitude rather than topographical data required closer study. The ANOVA with 9 electrodes from the region of interest confirmed an effect at fronto-central and mastoid electrodes (group $x$ electrode interaction: $\left.F_{8,304}=3.1, P=0.05, \varepsilon=0.24\right)$. Post-hoc t-tests at specific electrode sites confirmed that amplitudes were reduced in the older subjects at $\mathrm{FCz}$ and $\mathrm{Cz}$ by about $1 \mu \mathrm{V}(-2.0$ to -1.1 and -1.6 to $-0.6 \mu \mathrm{V}, \mathrm{t}(38)=-2.6$ to $-2.9, \mathrm{p}=0.01-0.006$, respectively). This trend was continued over the right hemisphere to $195 \mathrm{~ms}$ (Figure 1).

\section{Dipole Source Analysis}

The group solutions to the dipole locations and orientations are illustrated in Figure 2. As predicted, the wave forms in both groups were well explained by the four-dipole model of Jemel et al. (2002). Minor adjustments led to residual variances for group solutions below $1.8 \%$, and best fits below $0.7 \%$. Temporal lobe dipolesources on the left were on the borders of the superior temporal gyrus, insula and parietal cortex (BA 22, 29, 13). This is more dorso-medial and posterior to the temporal lobe source on the right (BA 21, 22, 42). The left frontal source lay posteriorly in the anterior cingulate (BA 24), while that on the right was further rostral and varied between adjacent regions of the inferior and mid-frontal cortices (BA 10, 45, 44). Table 1 presents the mean Talairach coordinates for the dipole sources for individuals in the two groups, and Table 2 shows the mean of the moments, latencies and orientation of each of these 4 dipoles for the individuals in each group.

As illustrated in Figure 2, the locations of the dipoles differed significantly between the adolescent and the young adult subjects $\left[F_{12}\right.$, ${ }_{22}=9.9, P<0.001$, Wilks $\Lambda=0.156$ ]. Left temporal lobe sources were 10-12 mm more ventro-lateral in the older subjects $\left(F_{s_{1}, 33}>19.8, P_{s}<0.001\right)$. In the right hemisphere temporal lobe sources were 8-14 mm more ventro-medial in the 30Y-group. In the frontal lobes the left cingular source was 13 $\mathrm{mm}$ more posterior $\left(F_{1,33}=26.6, P<0.001\right)$ but the right frontal source lay $10-14 \mathrm{~mm}$ more anteromedial in the older subjects $\left(F_{s_{1}},{ }_{33}>32.4\right.$, $P s<0.001)$. 


\section{Table 1}

The mean (and SD) of the individual values for the residual variance and best fit of the model and the Talairach coordinates for the locus defined by the mean

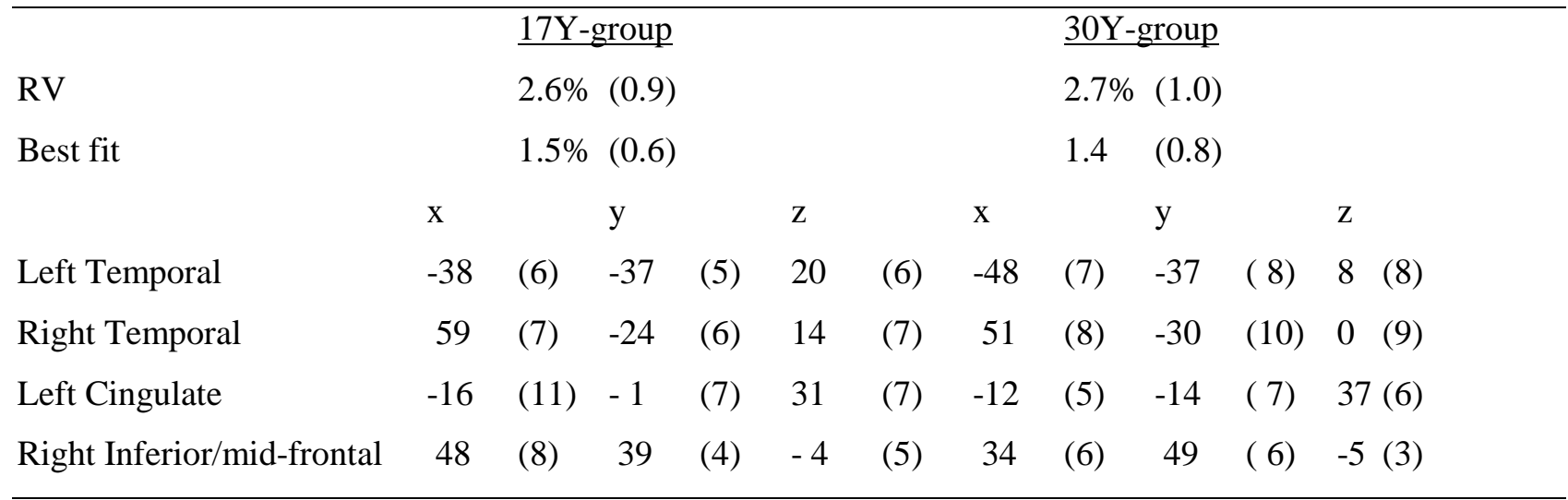

Table 2

Mean (and SD) of individual subjects' dipole strength, latency and orientation: adolescent vs young adult groups

\begin{tabular}{lccccccccc}
\hline & \multicolumn{4}{c}{ 17Y-group } & \multicolumn{5}{c}{ 30Y-group } \\
Dipole & $\begin{array}{l}\text { Strength } \\
(\mathrm{nA})\end{array}$ & $\begin{array}{l}\text { Latency } \\
(\mathrm{ms})\end{array}$ & $\begin{array}{l}\text { Orientation } \\
(\text { degrees })\end{array}$ & $\begin{array}{l}\text { Strength } \\
(\mathrm{nA})\end{array}$ & $\begin{array}{l}\text { Latency } \\
(\mathrm{ms})\end{array}$ & $\begin{array}{l}\text { Orientation } \\
(\text { degrees })\end{array}$ \\
& & & 0 & $\varphi$ & & & $\theta$ & $\varphi$ \\
\hline Left & 25.8 & 159.1 & -27.3 & 3.2 & 18.6 & 152.8 & -52.2 & 23.2 \\
$\quad$ Temporal & $(11.3)$ & $(20.1)$ & $(129)$ & $(54)$ & $(6.8)$ & $(20.7)$ & $(127)$ & $(50.9)$ \\
Right & 17.6 & 164.7 & -26.7 & 5.1 & 19.4 & 154.8 & -59.3 & 14.9 \\
$\quad$ Temporal & $(5.0)$ & $(20.3)$ & $(115)$ & $(66)$ & $(8.2)$ & $(22.7)$ & $(109)$ & $(66.7)$ \\
Left & 24.3 & 154.5 & 39.1 & -1.5 & 22.6 & 159.5 & 36.3 & -15.8 \\
$\quad$ Cingulate & $(13.4)$ & $(27.7)$ & $(114)$ & $(57)$ & $(16.3)$ & $(25.8)$ & $(101)$ & $(54.5)$ \\
Right & 15.9 & 164.7 & 51.8 & -28.7 & 15.4 & 177.1 & 91.8 & -15.9 \\
$\quad$ Inferior/mid-frontal & $(13.6)$ & $(31.0)$ & $(114)$ & $(58)$ & $(9.6)$ & $(24.0)$ & $(83.4)$ & $(57.0)$ \\
\hline
\end{tabular}

The peak latencies for each dipole moment were similar between groups. The 10-12 ms differences in the right hemisphere of older subjects (significant interaction: $F_{3,99}=3.4, P=$ $0.025)$ proved non-significant post-hoc ( $P \mathrm{~s}=0.18$ $0.21)$. But across groups the moment of the right frontal source $(170 \mathrm{~ms})$ peaked later than for the cingulate $(157 \mathrm{~ms})$ or the sources in the temporal lobes (left/right, 156/160 ms: right vs. left frontal dipoles $\mathrm{t}_{34}=-3.35, P<0.05$; vs. left temporal lobe $\left.\mathrm{t}_{34}=-2.68, P<0.10\right)$. This illustrates a bottom-up sequence of activation.

Similarly the strength of the dipole moments did not differ significantly between groups, although there were general differences between dipole sources $\left(F_{3,99}=4.77, P=0.008\right)$. The right frontal source (15.7 nA) was weaker than the left cingulate source $(23.6 \mathrm{nA})$ or those in the temporal lobes (left/right, 23.6/22.7 nA: right vs. left frontal dipoles $\mathrm{t}(34)=2.9, P<0.05)$, vs. left temporal dipole, $\mathrm{t}(34)=2.6, P<0.10)$. The data on dipole orientation are presented in table 2 .

\section{$M M N$ and Dipole Source Analysis in the Active Auditory Discrimination}

A decrease in the MMN amplitude with age, that resembled the reduction recorded 
Figure 3:

The figure illustrates the duration-deviant MMN waveforms for the $17 \mathrm{Y}$ group and the $30 \mathrm{Y}$ group of subjects recorded from 7 fronto-central and two mastoid electrode-sites during the active auditory condition

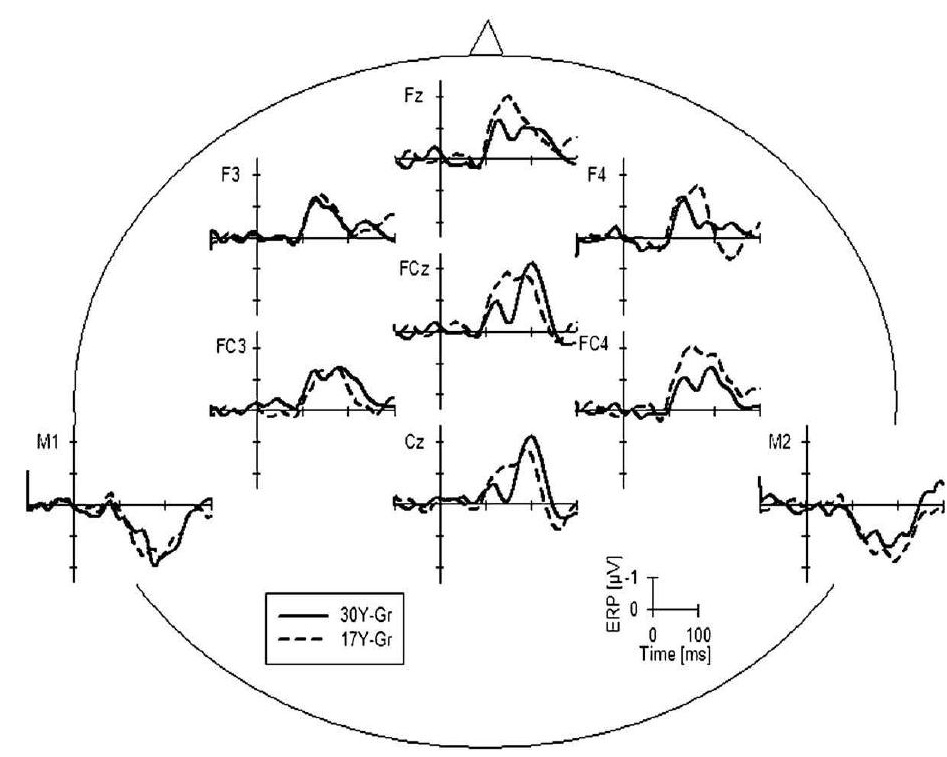

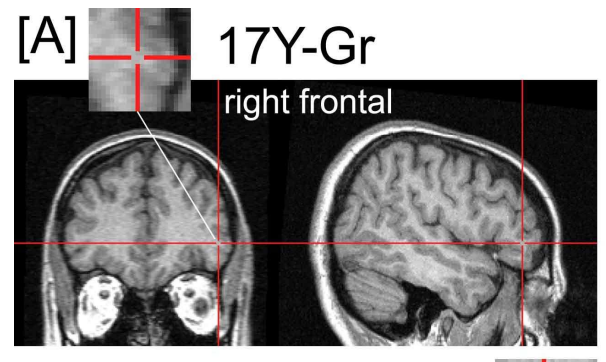
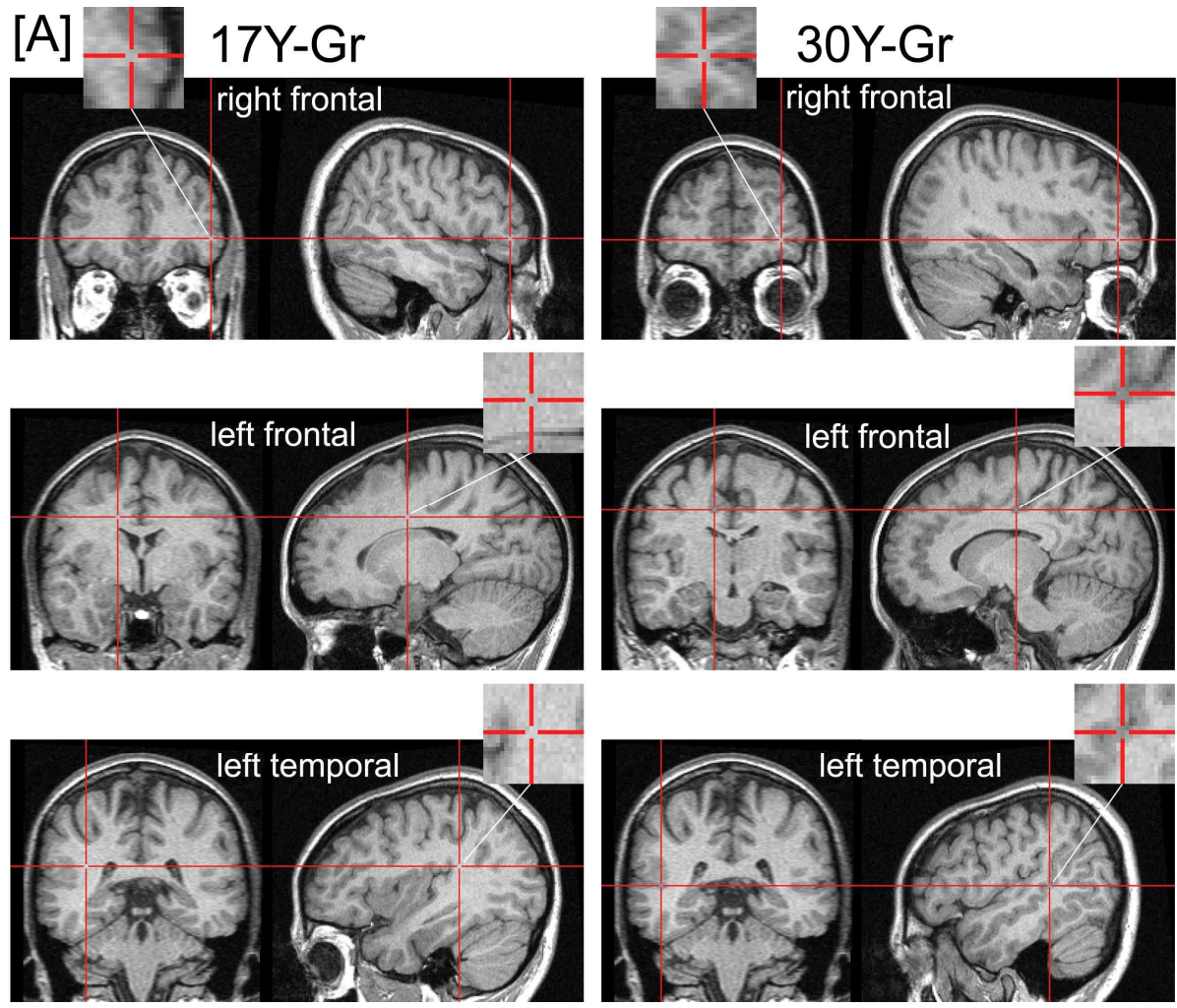

$[\mathrm{B}]$

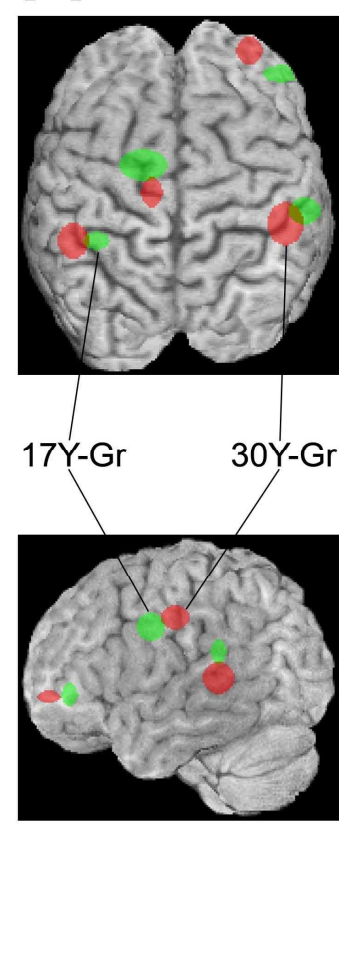

Figure 4: [A] Individual sagittal and axial MRI scans of an individual brain from the $17 \mathrm{Y}$ - and $30 \mathrm{Y}$ subject groups to illustrate the locus of the right inferior/mid-frontal left fronto-cingular and left temporal dipole sources. Inserts show a close-up of the calculated source-locus. [B] A view of the brain from above and the side with circles/ellipses (white $=17 \mathrm{Y}$, black $=30 \mathrm{Y}$ ) showing the locus for the group-solution and spread of the individual source-solutions of the subjects in each group. The radius in each dimension represents the standard deviation of the values for the constituent subjects.

in the passive condition, was confirmed for the active auditory condition requiring attention to auditory stimuli (Figure 4). This could be demonstrated in an analysis of 29 electrodes (group $x$ electrode interaction: $F_{28,1064}=3.99, P=$ $0.001, \varepsilon=0.21$ ) as well as for the 9 electrode regions-of-interest analysis for the 135 to $165 \mathrm{~ms}$ post-stimulus time window $\left(F_{8,304}=6.17, P=\right.$ $0.001, \varepsilon=0.36$ ). The analysis of the vector normed data in the interaction showed no significant differences. Post-hoc tests for the region-of-interest analysis revealed a reduced 
MMN amplitude for the older subjects at frontocentral electrode sites that was pronounced for the right hemisphere (for Fz, F4, FCz, FC4 and Cz: $\left.\mathrm{ts}(38)>2.4, P_{\mathrm{S}}<0.02\right)$. There was no significant interaction with group, but a comparison between conditions (condition $x$ electrode interaction) showed MMN to be larger in both groups in the passive condition (attention to visual stimuli) than in the active condition. This result related to both the $135-165 \mathrm{~ms}\left(F_{8,304}=19.2\right.$, $P<0.001, \varepsilon=0.35)$ and the $165-195$ ms time windows $\left(F_{8,304}=9.1, P<0.001, \varepsilon=0.36\right)$.

The group solutions for MMN dipoles in the passive condition were then fitted to the data from the active condition. An acceptable fit for location was obtained (17Y-group RV $=3.5 \%$; 30Ygroup $\mathrm{RV}=5.2 \%)$. This result was obtained with only minor changes to orientation that probably reflected trivial alterations in the degree of tissue contributing to the source. Thus, the locations of the neuronal generators for $\mathrm{MMN}$ were found to be comparable between conditions.

\section{Discussion}

In the first instance we have replicated the general dipole location model for $\mathrm{MMN}$ reported by Jemel et al. (2002) for change detection of the frequency of auditory tones, and extended it with source analysis to the detection of changes according to stimulus duration in adolescent and young adult subjects. Moderately asymmetric sources were located in the secondary auditory cortices on the left, and slightly more anteriorly in the right hemisphere. The left frontal source lay posteriorly in the anterior cingulate gyrus, while that on the right was found on the borders of the inferior- and mid-frontal cortices.

Secondly, there were important differences in the characteristics of measures of brain activity reflecting automatic processing of change detection (MMN) between teenagers (17Y-group) and young adults (30Y-group). MMN amplitudes were reduced by about a third at 9 fronto-central and mastoid sites in the older subjects, while N1 amplitudes were unchanged. The MMN decrease was reflected by changes of dipole source location. Unexpectedly, alterations of dipole peak moment (smaller in the left temporal lobe), latency (delayed in the right frontal cortex) or orientation did not significantly contribute to a group difference. But this probably reflects the more unstable values for the individual source solutions (necessary for the statistical analysis), that were derived from relatively few trials compared to the group solution. Generalising, with increasing age there were changes in the location of dipole sources on two different axes. In the temp-oral lobe sources became more (left) ventrally located. In the frontal lobe the distance between sources increased on the rostro-caudal axis, with posterior frontal sources more caudal on the left, and anterior frontal sources more rostral on the right.

These alterations obtain significance firstly in view of a low awareness amongst practitioners that brain structure and function continue to develop through the third decade of life during which psychiatric illness may start (e.g. psychosis) or alter its manifestation (e.g. adult $A D / H D$ ). Secondly, the role of MMN may be taken as a proxy for the functionality of early and automatic selective information processing. Such processes that have their origin in association cortices (temporal lobe, the cingulate and right inferior/mid-frontal cortices) become important candidate markers for poor function, as is particularly evident in developing and adult cases of $A D / H D$.

The right inferior/mid-frontal location is broadly consistent with neuroimaging data implicating this region in auditory attention processes (Paus, 2002), and in generic 'change detection' (Beck et al., 2001). The particular function has been described as the initiation of a switch in processing information channels appropriate (and adaptive) to the perceived change in the stimulation (Escera et al., 2000). The contribution of the cingulate source to change detection is unclear, but may be related to conflict monitoring for change and error detection (Carter et al., 1998; Brazdil et al., 2002). ERP and imaging studies of trial and error learning (Holroyd et al., 2004) showed that both making mistakes, watching mistakes being made (Van Schie et al., 2004) and obtaining feedback activated the posterior-dorsal anterior cingulate close to the area we report to contribute to MMN. The authors suggest that this region is sensitive to both internal and external sources of error information. We propose that there is a perceptual feature that can be described as "a deviation" that is common to the mismatch involved in error detection and the mismatch noted by a change of stimulus in a series of similar stimuli. Monitoring the occurrence of both sorts of deviations by the cingulate region would 
be efficient because of the connectivity of the region, and adaptive in view of the potential consequences of the change.

\section{The MMN Model:}

How reliable are the source locations reported? Frontal and temporal source activity has also been illustrated by Valkonen-Korhonen et al. (2003), although they did not attempt precise localisation. MMN sources have been reported for association areas in the superior temporal gyrus and anterior cingulate gyrus by Waberski et al. (2001), although unlike our study, their cingulate source was much weaker than those of the temporal lobe. Further it peaked considerably later, and may have reflected contributions from mid-frontal activity. We have observed a similar "smearing" of fronto-cingulate activity in a trial examination of MMN waveforms that sometimes appear with a double peak using Loreta source analysis methods (unpublished data).

The precision of the calculation of the dipole location is increased by the digitization of the recording sites with respect to individual fiducial skull markers. Nonetheless the solution provided is a point in the grey-matter. Physiologically speaking this is implausible: it is likely that the active tissue will extend over a larger area. Earlier we reported a dipole analysis checking the stability of the solution over successive millimetres away from the point solution (Jemel et al., 2003). We found the solution usually to be stable over 2-6 $\mathrm{mm}$ along each dimension. Further, the variability between subjects in the present study, despite the poor signal-to-noise ratio was usually well below a centimetre (Table 1). This upper limit for the accuracy of the model matches well that obtained from known modelled sources (Yvert et al., 1997; Laarne et al., 2000). But, in addition to these indicators of intra-group and inter-individual stability of the model, error is introduced in the placing of sources on the brain atlas or SPM maps based on the anatomy derived from averages of other subjects. In part we have corrected for this by representing sources on the anatomy of one of our subjects (figure 3). But without construction of an average brain image for our subjects, we have not accounted for the putative anatomical variability within our subjects.

The reliability of the calculated model also depends on the recorded signal-to-noise ratio.
Evidence suggests that a reasonable effect size requires data for 6 electrodes per dipole from a thousand artefact-free trials (Kamijo et al., 2002; Phillips et al., 2002; Picton et al., 1995). While this requirement was satisfied here for the group solution, it must be appreciated that it would not be possible with our recording configuration to resolve separate dipoles in, say, the primary and secondary auditory cortices (cf. Jääskeläinen et al., 2004). Nonetheless, the stability of the group solution that accounted for over $98 \%$ of the variance in the data was aided by the hypothesisled model (Jemel et al., 2002). For the individual solutions, that provided a measure of variance between subjects, we still found that data from $20 / 22$ subjects in the $17 Y$ - and $15 / 18$ subjects in the $30 Y$-groups explained $96 \%$ of the variance in this MMN model.

\section{Structure and Function:}

What do changes in the coordinates of the MMN dipoles with age represent? It is oversimplified to attribute the changes to a straightforward increase in volume for two reasons. First, the changes in both frontal and temporal lobe loci take place in two dimensions. The increase did not merely reflect volume (3 dimensions) or a rostro-caudal extension (one dimension). Second, the axes of change in the frontal and temporal lobes differ from each other. In both temporal lobes the change with age was ventral and left, while in the left cingulate it was posterior (and dorsal) contrasting with the anterior (and medial) change in the inferior/mid frontal location. Such changes could represent the final maturational stage of 'torque', whereby the lateralised asymmetric increase in volume of the frontal lobe develops on the right, and that in the temporal lobe develops on the left (Mackay et al., 2003).

Could torque be affected in $A D / H D$ where brain size around puberty is modestly reduced (Castellanos et al., 1996)? Apparently torque development does not relate to brain size (Mackay et al., 2003). But a focus on this developmental feature as a potential impairment in adult $A D / H D$ should form a basis for future study in view of Durston and colleagues' findings (2004) of 4-9\% reductions in grey matter (and to a lesser extent white matter) in the right frontal and left occipital regions of the brain of $A D / H D$ subjects at the time of puberty. Castellanos and colleagues (2002) also noted not only reduced 
white matter in childhood $A D / H D$, but reduced cerebral grey matter in frontal and temporal cortices that was correlated with the severity of psychopathology. Filipek (1999) noted that the involvement of right frontal regions in particular seemed to depend on comorbid problems. Indeed, a recent MRI study of adolescents with conduct disorder (independent of substanceabuse) showed volumetric reductions in the right hemisphere, reminiscent of $A D / H D$ studies (Kruesi et al., 2004). But, in contrast to AD/HD studies the emphasis was on reductions in the right temporal lobe, while those in the right frontal lobe did not attain significance. This is an intriguing subtle difference between the associations of two disorders that are often comorbid.

This leads to an initial prediction. If $A D / H D$ manifests in an adult with marked comorbid conduct problems, then structural and functional characteristics may be more related to temporal than frontal lobe anomalies. This could result in problems of working memory and concentration rather than restlessness and impulsivity. The reasoning here is that it is in the frontal lobes that a lack of activation is apparent in adolescent $A D / H D$ subjects required to inhibit responses (Rubia et al. in press). If the lack of a lateral migration has occurred as documented in the present study then an immature level of temporal lobe function is to be expected.

However, the apparent maturational changes in MMN source and functional localization might relate to more specific and localized anatomical development. Sowell and colleagues (2001) have shown that there are not only late changes in gyral surface area and grey/white matter ratios, but especially growth and grey matter density reductions in the region of the temporal-occipital junction and inferior frontal cortex occurring at the end of normal development. In these regions grey matter may decrease linearly while white matter develops quadratically to the end of the fourth decade (Bartzokis et al., 2001). But do the results of our localization study reflect local uneven rates of development and expansion between adjacent regions of the brain or a change in the nature of cortical substance active in generating MMN? Close inspection of the represent-ations in figure 3 leads to equivocal conclusions. The change in the right inferior frontal source would be consistent with a local expansion, while the left cingular source appears to have migrated around a mid-cingular lobule, potentially staying within the same functional region. In contrast, the left temporal source appears to have moved to a separate more ventral locus. Such suggestions are clearly tentative and speculative. The sources represent averages of the data from several persons in the group, and the anatomy concerns only one of them. But the suggestions serve as predictions that can be tested in the sort of prospective study run by Gogtay et al. (2004).

In particular, if we ascribe right hemispheric, and in particular right frontal features of $A D / H D$ to a lag in development (with MMN sources as the marker), then only adult cases of $A D / H D$ with a history of adolescent and childhood AD/HD would be expected to retain the wide-ranging clinical picture: the right frontal rostral extension did not take place. In cases where the clinical picture has arisen de novo, or is partial in extent either development has partly caught up (a possibility with a third decade of post-adolescent development to be expected) or, more likely, the $A D / H D$ symptoms have a separate origin. In both cases the prognosis should be better, for either the separate cause may be capable of isolation and treatment or there is a neurobiological basis for training or medical treatment to take effect. It may be noted here that we are advocating that future adult $A D / H D$ testing should make use of such sub-grouping that might otherwise disappear through combining potential categories of patient and comparing them merely with age-matched comparison subjects. In addition, in this way use of the MMN source as a structural-functional marker may be applied as a test of the developmental lag hypothesis.

From a functional viewpoint, and bearing in mind future studies of structural-functional relationships in psychiatric patients, it is important to note that we are not describing an impairment in this report. A significant reduction of MMN amplitude (not reflected in the strength of the dipole moment) was associated with a significant change of the coordinates for the underlying sources. It must be presumed that, whether this reflects a change of anatomical locus or detailed changes in grey-white matter relationships (and associated conductivity), the result is presumably a change of efficiency in automatic information selection (auditory change detection). This is an intriguing change of the baseline in normal function associated with post- 
adolescent maturation in the third decade of life. This emphasizes the care with which psychopathological studies of ERPs and the structures generating them need to be interpreted.

\section{References}

Aron, A.R., Fletcher, P.C., Bullmore, E.T., Sahakian, B.J., Robbins, T.W., 2003. Stopsignal inhibition disrupted by damage to right inferior frontal gyrus in humans. Nature Neurosci., 6, 115-116.

Barry, R.J., Johnstone, S.J., Clarke, A.R., 2003. A review of electrophysiology in attentiondeficit/hyperactivity disorder: II Event-related potentials. Clin. Neurophysiol., 114, 184-198.

Bartzokis, G., Meckson, M., Lu, P.H., Nuechterlein, K.H., Edwards, N., Mintz, J., 2001. Age-related changes in frontal and temporal lobe volumes in men: a magnetic resonance imaging study. Arch. Gen. Psychiatry, 58, 461-465.

Beck, D.M., Rees, G., Frith, C.D., Lavie, N., 2001. Neural correlates of change detection and change blindness. Nature Neurosci. 4, 645650.

Benes, F.M., Turtle, M., Farol, P., 1994. Myelination of a key relay zone in the hippocampal formation occurs in the human brain during childhood, adolescence and adulthood. Arch. Gen. Psychiatry, 51, 477-484.

Biederman, J., Mick, E., Faraone, S.V., 2000. Agedependent decline of symptoms of attention deficit hyperactivity disorder: impact of remission definition and symptom type. Am. J. Psychiatry, 157, 816-818.

Blinkov, S.M., Glezer, I.I., 1968. The human brain in figures and tables: a quantitative handbook. Plenum Press: New York.

Blyler, C.R., Gold, J.M., lannone, V.N., Buchanan, R.W., 2000. Short form of the WAIS-III for use with patients with schizophrenia. Schizophr. Res., 46, 209-215.

Brazdil, M., Roman, R., Falkenstein, M., Daniel, P., Jurak, P., Rektor, I., 2002. Error processingevidence from intra-cerebral ERP recordings. Exp. Brain Res., 146, 460-466.

Carter, C.S., Braver, T.S., Barch, D.M., Botvinick, M.W., Noll, D., Cohen, J.D., 1998. Anterior cingulate cortex, error detection and the online monitoring of performance. Science 280, 274-279.
Casey, B.J., Trainor, R., Giedd, J.N., Vauss, Y., Vaituzis, C.K., Hamburger, S.D. et al., 1997. The role of the anterior cingulate in automatic and controlled pro-cesses: a developmental neuroanatomical study. Dev. Psychobiol., 30, 61-69.

Castellanos, F.X., Giedd, J.N., Marsh, W.L., Hamburger, S.D., Vaituzis, A.C., Dickstein, D.P. et al., 1996. Quantitative brain magnetic resonance imaging in attention-deficit hyperactivity disorder. Arch. Gen. Psychiatry, 53, 607-616.

Castellanos, F.X., Lee, P.P., Sharp, W., Jeffries, N.O., Greenstein, D.K., Clasen, L.S. et al., 2002. Developmental trajectories of brain volume abnormalities in children and adolescents with attention-deficit/hyperactivity disorder. J. Am. Med. Assocn., 288, 1740-1748.

Corbett, B., Stanczak, D.E., 1999. Neuropsychological performance of adults evidencing attention-deficit hyperactivity disorder. Arch. Clin. Neuropsychol., 14, 373387.

Crottaz-Herbette, S., Ragot, R., 2000. Perception of complex sounds: N1 latency codes pitch and topography codes spectra. Electroencephalogr. Clin. Neurophysiol. 111, 1759-1766.

Duffy, F.H., Albert, M.S., McAnulty, G. Garvey, A.J., 1984. Age-related differences in brain electrical activity of healthy subjects. Ann. Neurol., 16, 430-438.

Durston, S., Hulshoff Pol, H.E., Schnack, H.G., Buitelaar, J., Steenhuis, M.P., Minderaa, R.B. et al., 2004. Magnetic resonance imaging of boys with attention-deficit/hyperactivity disorder \& their unaffected siblings. J. Am. Acad. Child Adolesc. Psychiatry, 43, 332-340.

Erikson, R., Goldthorpe, J.H., 1992. Individual or family? Results from two approaches to class assignment. Acta Sociol., 35, 95-106.

Escera, C., Alho, K., Schröger, E., Winkler, I., 2000. Involuntary attention and distractibility as evaluated with event-related potentials. Audiol. Neurootol., 5, 151-166.

Filipek, P.A., 1999. Neuroimaging in the developmental disorders: the state of the science. J. Child Psychol. Psychiatry., 40, 113128.

Giedd, J.N., 1997. Normal development. Child Adolesc. Psychiatry. Clin. N. Am., 6, 265-282.

Gogtay, N., Giedd, J.N., Lusk, L., Hayashi, K.M., Greenstein, D., Vaituzis, A.C. et al., 2004. Dynamic mapping of human cortical 
development during childhood through early adulthood. Proc. Natl. Acad. Sci. (U.S.A), 101, 8174-8179.

Goldman-Rakic, P., 1987. Development of cortical circuitry and cognitive function. Child Dev., 58, 601-622.

Harris, L.R., Lieberman, L., 1996. Auditory stimulus detection is not suppressed during saccadic eye movements. Perception, 25, 9991004.

Hendren, R.L., 1991. Psychotherapy of adolescents with attention-deficit disorder. In: Slomowitz, M. (Eds.), Adolescent Psychotherapy. 125-142, American Psychiatric Press: Washington.

Holroyd, C.B., Nieuwenhuis, S., Yeung, N., Nystrom, L.E., Mars, R.B., Coles, M.G.H., Cohen, J.D., 2004. Dorsal anterior cingulate cortex shows $\mathrm{fMRI}$ response to internal and external error signals. Nature Neurosci., 7, 497-498.

Jääskeläinen, I.P., Ahveninen, J., Bonmassar, G., Dale, A.M., Ilmoniemi, R.J., Levänen, S. et al., (2004). Human posterior auditory cortex gates novel sounds to consciousness. Proc. Natl. Acad. Sci. (U.S.A.), 101, 6809-6814.

Jemel, B., Achenbach, C., Müller, B., Röpcke, B., Oades, R.D., 2002. Mismatch negativity results from bilateral asymmetric dipole sources in the frontal and temporal lobes. Brain Topogr., $15,13-27$.

Jemel, B., Oades, R.D., Oknina, L., Achenbach, C., Röpcke, B., 2003. Frontal and temporal lobe sources for a marker of controlled auditory attention: the negative difference $(\mathrm{Nd})$ eventrelated potential. Brain Topogr., 15, 249-262.

Johnstone, S.J., Barry, R.J., 1996. Auditory eventrelated potentials to a two-tone discrimination paradigm in attention deficithyperactivity disorder. Psychiatry. Res., 64, 179-192.

Johnstone, S.J., Barry, R.J., Anderson, J.W., Coyle, S.F., 1996. Age-related changes in child and adolescent event-related potential component morphology, amplitude and latency to standard and target stimuli in an auditory oddball task. Int. J. Psychophysiol., 24, 223238.

Jückel, G., Gallinat, J., Riedel, M., Sokullu, S., Schulz, C., Möller, H-J. et al., 2003. Serotonergic dysfunction in schizophrenia assessed by the loudness dependence measure of primary auditory cortex evoked activity. Schizophr. Res., 64, 115-124.
Kalsbeek, A., 1989. The role of dopamine in the development of the rat prefrontal cortex. Krips Repro meppel (Acad. proefschrift): Amsterdam.

Kemner, C., Jonkman, L.M., Kenemans, J.L., Böcker, K.B.E., Verbaten, M.N. van Engeland, H., 2004. Sources of auditory selective attention and the effects of methylphenidate in children with attention-deficit/hyperactivity disorder. Biol. Psychiatry, 55, 776-778.

Kemner, C., Verbaten, M.N., Koelega, H.S., Buitelaar, J.K., van der Gaag, R.J., Camfferman, G., van Engeland, H., 1996. Event-related brain potentials in children with attention-deficit and hyperactivity disorder: effects of stimulus deviancy and task relevance in the visual and auditory modality. Biol. Psychiatry, 40, 522534.

Kamijo, K-I., Yamazaki, T., Kiyuna, T., Takaki, Y., Kuroiwa, Y., 2002. Visual event-related potentials during movement imagery and the dipole analysis. Brain Topogr., 14, 279-292.

Kruesi, M.J.P., Casanova, M.F., Mannheim, G., Johnson-Bilder, A., 2004. Reduced temporallobe volume in early onset conduct disorderPsychiatry. Res. (Neuroimaging), 132, 1-11.

Laarne, P.H., Tenhunen-Eskelinen, M.L., Hyttinen, J.K., Eskola, H.J., 2000. Effect of EEG electrode density on dipole localization accuracy using two realistically shaped skull resistivity models. Brain Topogr., 12, 249-254.

Lange, J.J., Wijers, A.A., Mulder, J.M., Mulder, G., 1998. Color selection and location selection in ERPs: differences, similarities and neural specificity. Biol. Psychol., 48, 153-82.

Lazzaro, I.; Whitmont, G.E.; Meares, R.; Clarke, S., 2001. The modulation of late component event related potentials by pre-stimulus EEG theta activity in ADHD. Int. J. Neurosci., 107, 247-264.

Lewis, D.A., Anderson, S.A., Rosenberg, D.R., Lund, J.S., Conde, F., 1995. Postnatal refinements of prefrontal circuitry and schizophrenia. Schizophr. Res., 15, 29.

Libet, B., 2002. The timing of mental events: Libet's experimental findings and their implications. Consciousness Cogn., 11, 291299.

Mackay, C.E., Barrick, T.R., Roberts, N. Delisi, L.E., Maes, F., Vandermeulen, D. et al., 2003. Application of a new image analysis technique to study brain asymmetry in schizophrenia. Psychiatry. Res. Neuroimaging, 124, 23-35. 
Matousek, N., Petersen, I., 1973. Frequency analysis of the EEG in normal children and adolescents. In, Kellaway, P. Petersen, I., Automation of Clinical Electroencephalography, Raven Press, New York, pp 75-102.

Mathalon, D.H., Whitfield, S.L., Ford, J.M., 2003. Anatomy of an error: ERP and fMRI. Biol. Psychol., 64, 119-141.

McCarthy, G., Wood, C.C., 1985. Scalp distributions of event-related potentials: an ambiguity associated with analysis of variance models. Electroencephalogr. Clin. Neurophysiol., 63, 203-208.

Murphy, P., 2002. Inhibitory Control in Adults with Attention-Deficit/Hyperactivity Disorder. J. Atten. Disord., 6, 1-4.

Oades, R.D., 1998. Frontal, temporal and lateralized brain function in children with attention-deficit hyperactivity-disorder: a psychophysiological and neuropsychological viewpoint on development. Behav. Brain Res., 94, 83-95.

Oades, R.D., Dittmann-Balcar, A., Zerbin, D., 1997. Development and topography of auditory event-related potentials, mismatch and processing negativity from 8 to 22 years of age. Psychophysiology, 34, 677-693.

Oades, R.D., Dittmann-Balcar, A., Schepker, R., Eggers, C., 1996. Auditory event-related potentials and mismatch negativity in healthy children and those with attention-deficit- or Tourette-like symptoms. Biol. Psychol., 43, 163-185.

Paus, T., 2002. Functional anatomy of arousal and attention systems in human brain. Prog. Brain Res., 126, 65-77.

Phillips, C., Rugg, M.D., Friston, K.J., 2002. Systematic regularization of linear inverse solutions of the EEG source localization problem. Neuroimage, 17, 287-301.

Pivik, R.T., Broughton, R.J., Coppola, R., Davidson, R.J., Fox, N., Nuwer, M.R., 1993. Guidelines for the recording and quantitative analysis of electroencephalographic activity in research contexts. Psychophysiology, 30, 547-558.

Picton, T.W., Lins, O.G., Scherg, M., 1995. The recording and analysis of event-related potentials. In: Boller, F., Grafman, J. (Eds.), Handbook of Neuropsychology, Amsterdam: Elsevier, pp 3-73.

Pliszka, S.R., Liotti, M., Woldorff, M.G., 2000. Inhibitory control in children with attentiondeficit/hyperactivity disorder: event-related potentials identify the processing component and timing of an impaired right-frontal response-inhibition mechanism. Biol. Psychiatry, 48, 238-246.

Rabinowicz, T., 1986. The differentiated maturation of the cerebral cortex. In: Falkner, F., Tanner, J.M. (Eds.), Human Growth: volume 2, Plenum Press, New York, pp. 385-410.

Ritter, W., Simson, R., Vaughan, H.G., 1983. Event-related potentials and two sequential stages of information processing in physical and semantic discrimination. Psychophysiology, 20, 168-179.

Rosenberg, D.R., Lewis, D.A., 1994. Changes in the dopaminergic innervation of monkey prefrontal cortex during late postnatal development: a tyrosine hydroxylase immunohistochemical study. Biol. Psychiatry, 36, 272-277.

Rothenberger, A., Banaschewski, T., Heinrich, H., Moll, G.H., Schmidt, M.H., van't Klooster, B., 2000. Comorbidity in ADHD-children: effects of co-existing conduct disorder or tic disorder on event-related brain potentials in an auditory selective-attention task. Eur. Arch. Psychiatry. Clin. Neurosci., 250,101-110.

Rubia, K., Taylor, E.A., Smith, A.B., Oksannen, H., Overmeyer, S., Newman, S., 2001. Neuropsychological analyses of impulsiveness in childhood hyperactivity. Br. J. Psychiatry, 179, 138-143.

Rubia, K., Smith, A.B., Brammer, M.J., Toone, B.K., Taylor, E.A., 2004. Medication-naive adolescents with attention-deficit hyperactivity disorder show abnormal brain activation during inhibition and error detection. Am. J. Psychiatry, in press.

Scherg, M., Berg, P., 1991. Use of a priori knowledge in brain electromagnetic brain activity. Brain Topogr. 4, 143-150.

Scherg, M., Picton, T.W., 1991. Separation and identification of event-related brain potential components by brain electric source analysis. Electroencephalogr. Clin. Neurophysiol. Suppl. 42, 24-37.

Schneider, W.; Shiffrin, R.M., 1977. Controlled and automatic human information processing: I. Detection, search and attention. Psychol. Rev., 84, 1-66.

Sowell, E.R., Thompson, P.M., Tessner, K.D., Toga, A.W., 2001. Mapping continued brain growth and gray matter density reduction in dorsal frontal cortex: Inverse relationships during 
post-adolescent brain maturation. J. Neurosci., 21, 8819-8829.

Thatcher, R. W., 1987. Maturation of the human frontal lobes. Physiological evidence for staging. Dev. Neuropsychol., 7, 397-419.

Treisman, A. M., (1969). Strategies and models of selective attention. Psychol. Rev., 76, 282-299.

Unis, A.S., Vincent, J.G., Dillon, B., 1995. Dopamine D2 receptor autoradiography with (3H) YM 09151-2 reflects brain and behavioral ontogeny. Prog. Neuropsychopharmacol. Biol. Psychiatry, 19, 333-342.

Valkonen-Korhonen, M., Purhonen, M., Tarkka, I.M., Sipilä, P., Partanen, J., Karhu, J. et al., 2003. Altered auditory consciousness in acutely psychotic, never-medicated firstepisode patients. Cogn. Brain Res. 17, 747758.

van Schie, H.T., Mars, R.B., Coles, M.G.H., Bekkering, H., 2004. Modulation of activity in medial frontal and motor cortices during error observation. Nature Neurosci., 7, 549-554.

Waberski, T.D., Kreitschmann-Andermahr, I., Kawohl, W., Darvas, F., Ryang, Y., Gobbele, R., Buchner, H., 2001. Spatio-temporal source imaging reveals subcomponents of the human auditory mismatch negativity in the cingulum and right inferior temporal gyrus. Neurosci. Lett., 308, 107-110.

Willoughby, M.T., 2003. Developmental course of ADHD symptomatology during the transition from childhood to adolescence: a review with recommendations. J. Child Psychol. Psychiatry. 44, 88-106.

Winsberg, B.G., Javitt, D.C., Shanahan-Silipo, G., 1997. Electrophysiological indices of information processing in methylphenidate responders. Biol. Psychiatry, 42, 434-445.

Yakovlev, P.I., Lecours, A., 1967. The myelinogenetic cycles of regional maturation of the brain. In: Minkowski, A. (Eds.), Regional development of the brain early in life, pp. 370, Blackwell: Oxford.

Yvert, B., Bertrand, O., Thevenet, M., Echallier, J.F., Pernier, J.A., 1997. Systematic evaluation of the spherical model accuracy in EEG dipole localization. Electroencephalogr. Clin. Neurophysiol., 102, 452-459. 